\title{
ESTIMATION THEORY FOR GROWTH AND IMMIGRATION RATES IN A MULTIPLICATIVE PROCESS
}

\author{
C. C. HEYDE AND \\ E. SENETA, Australian National University
}

\begin{abstract}
This paper deals with the simple Galton-Watson process with immigration, $\left\{X_{n}\right\}$ with offspring probability generating function (p.g.f.) $F(s)$ and immigration p.g.f. $B(s)$, under the basic assumption that the process is subcritical $\left(0<m \equiv F^{\prime}(1-)<1\right)$, and that $0<\lambda \equiv B^{\prime}(1-)<\infty, 0<B(0)<1$, together with various other moment assumptions as needed. Estimation theory for the rates $m$ and $\lambda$ on the basis of a single terminated realization of the process $\left\{X_{n}\right\}$ is developed, in that (strongly) consistent estimators for both $m$ and $\lambda$ are obtained, together with associated central limit theorems in relation to $m$ and $\mu \equiv \lambda(1-m)^{-1}$ Following this, historical antecedents are analysed, and some examples of application of the estimation theory are discussed, with particular reference to the continuous-time branching process with immigration. The paper also contains a strong law for martingales; and discusses relation of the above theory to that of a first order autoregressive process.

ESTIMATION THEORY; GROWTH AND IMMIGRATION RATES; MULTIPLICATIVE PROCESSES; STRONG CONSISTENCY; STRONG LAW; MARTINGALES; CENTRAL LIMIT THEOREM; FIRST ORDER AUTOREGRESSION; PARTICLE FLUCTUATION
\end{abstract}

\section{Introduction}

The fundamental process with which we shall be concerned is the simple Galton-Watson process with immigration, $\left\{X_{n}\right\}$, with offspring probability generating function (p.g.f.) $F(s)$, and immigration p.g.f. $B(s)$. In the present paper we confine ourselves to the subcritical case $\left(0<m \equiv F^{\prime}(1-)<1\right)$, assuming throughout also that $\lambda \equiv B^{\prime}(1-)<\infty, 0<B(0)<1$, and that the respective variances $\sigma^{2}$ and $b^{2}$ of the offspring and immigration distributions are finite. We do not make one of the usual assumptions that $X_{0}=1$ or $X_{0}=0$, but allow a general distribution, which, however, is assumed to satisfy $E\left(X_{0}^{2}\right)<\infty$ throughout. (Additional moment assumptions are made as needed.)

Our main aim in this paper is to develop an estimation theory for the rates $m$ and $\lambda$, on the basis of a single realization of the process $\left\{X_{n}\right\}$ (observed up to some fixed time).

The process, under the present assumptions, is not stationary in general, so

Received 25 June 1971. 
it is not possible to avail oneself directly of the large body of estimation theory known for such processes. However, it is known (Heathcote (1966); Seneta (1969), Section 5.2) that the process approaches a limiting-stationary distribution as $n \rightarrow \infty$, so it is not unexpected that our results resemble known ones for stationary regimes.

In the same vein, the present paper considers techniques which (in the usual manner of time-series analysis) attempt to exploit the totality of information contained in a realization of the process. Another approach, based more on recurrence properties of individual states, is possible and in some ways more general and convenient. It does, however, seem to have the disadvantage of not utilizing all the available information, and so will be discussed in a sequel to this paper. This sequel will also contain estimation theory for $\lambda$ in the critical case $m=1$; and for the individual variances $\sigma^{2}$ and $b^{2}$.

Following the development of the general estimation theory in the present paper, we analyse its historical antecedents, and discuss some examples of its application, with particular reference to the continuous-time branching process with immigration.

In conclusion to this introduction, we note that in the supercritical $(1<m<\infty)$ case for both the processes with and without immigration, results for the estimation of $m$ are already available (Heyde (1971); Heyde and Seneta (1971)).

\section{Statement of main results and discussion}

Theorem A. Let $C_{n}=\sum_{i=1}^{n} X_{i} / n(\equiv \bar{X})$ and $D_{n}=\sum_{i=1}^{n} \hat{Y}_{i+1} \hat{Y}_{i} / \sum_{i=1}^{n} \hat{Y}_{i}^{2}$, where $\hat{Y}_{i}=X_{i}-C_{n}$. Then $C_{n} \stackrel{\stackrel{p}{\rightarrow}}{\mu} \equiv \lambda(1-m)^{-1}$, and $D_{n} \stackrel{\mathfrak{p}}{\rightarrow} m$ as $n \rightarrow \infty$, so that $C_{n}\left(1-D_{n}\right)$ and $D_{n}$ are consistent estimators of $\lambda$ and $m$ respectively. If the offspring, immigration and initial distributions all have finite third moments, then the above convergences in probability can be replaced by almost sure convergence. That is, the respective estimators are strongly consistent.

It will also be shown in the sequel that, in the above theorem, it is possible to use the statistic

$$
D_{n}^{*}=1-\frac{1}{2} \frac{\sum_{i=1}^{n}\left(X_{i+1}-X_{i}\right)^{2}}{\sum_{i=1}^{n}\left(X_{i}-\bar{X}\right)^{2}}
$$

in place of $D_{n}, D_{n}^{*}$ being possibly more convenient computationally and aesthetically.

The other main result is the following theorem, which provides an indication of the convergence rates of $C_{n}$ and $D_{n}$ to their respective limits, in the form of central-limit type results. It thus makes possible statistical inferences on the offspring mean, $m$, and the mean, $\mu$, of the limiting-stationary distribution, on the basis of a single long realization of $\left\{X_{n}\right\}$. 
For purposes of this theorem, and the sequel in general, we introduce the notation $c^{2}$ for the frequently occurring quantity $b^{2}+\sigma^{2} \mu$.

Theorem B.

$$
\lim _{n \rightarrow \infty} P\left(\alpha^{-1} \sqrt{ } n\left(C_{n}-\mu\right) \leqq x\right)=\Phi(x),
$$

where $\alpha^{2}=(1-m)^{-2} c^{2}$, and $\Phi(x)=(2 \pi)^{-1 / 2} \int_{-\infty}^{x} e^{-u^{2} / 2} d u$.

If, in addition to the basic assumptions of Section 1, all of the offspring, immigration, and initial distributions have finite fourth moments ${ }^{1}$, then

$$
\lim _{n \rightarrow \infty} P\left(\beta^{-1} \sqrt{ } n\left(D_{n}-m\right) \leqq x\right)=\Phi(x)
$$

when $\beta=B\left(1-m^{2}\right) c^{-2}$. Here

$B=\left(1-m^{2}\right)^{-1} c^{4}+\sigma^{3}(1-m)^{-3}\left\{E(I-\lambda)^{3}+\mu E\left(Z_{1}-m\right)^{3}+3 m \sigma^{2}\left(1-m^{2}\right)^{-1} c^{2}\right\}$,

where $I$ and $Z_{1}$ are random variables, respectively having the distributions of the immigration and offspring mechanisms.

In this theorem also, $D_{n}$ may be replaced by $D_{n}^{*}$. The nature of the above results and of the statistics entering into them may readily be understood by comparison with another, simpler (time-series) context. To this end we first note that for our process $\left\{X_{n}\right\}$

$$
E\left(X_{n+1} \mid X_{n}\right)=m X_{n}+\lambda, \quad n=0,1,2, \cdots
$$

so that if we introduce the 'limit-mean corrected' process $\left\{Y_{n}\right\}$ where $Y_{n}=X_{n}-\mu$, $\mu \equiv \lambda(1-m)^{-1}$ being the mean of the limiting-stationary distribution of $\left\{X_{n}\right\}$, we have

$$
E\left(Y_{n+1} \mid Y_{n}\right)=m Y_{n}
$$

so that

$$
Y_{n+1}=m Y_{n}+\delta_{n}, \quad n=0,1,2, \cdots
$$

where $E\left(\delta_{n}\right)=0$ and (the residual) $\delta_{n}$ is uncorrelated with $Y_{n}$.

The relation (2.3) is similar to the defining relation

$$
x_{n+1}=\rho x_{n}+\varepsilon_{n}, \quad n=0, \pm 1, \pm 2, \cdots(|\rho|<1)
$$

of the second-order stationary process which is called a first-order autoregression, $\left\{\varepsilon_{n}\right\}$ being a sequence of independent and identically distributed normal variables

1 It is likely that this condition can be weakened to finiteness of third moments. 
with zero mean (i.e., 'white noise'). (For this process $\rho$ is the first autocorrelation.) Thus in our process $m$ (roughly) corresponds to $\rho$, and it is easily checked that our estimators $C_{n}$ and $D_{n}$ are analogous in form to the large sample maximum likelihood estimators of the corresponding quantities in (2.4), $C_{n}$ being the sample mean, and $D_{n}$ effectively the first circular serial correlation coefficient, while $D_{n}^{*}$ corresponds to the correlation statistic $r_{A}$ related to Von Neumann's ratio (see Hannan (1960), pp. 84-85).

Whereas our process $\left\{X_{n}\right\}$ is far, in general, from satisfying the conditions of the first-order autoregression as regards stationarity, and normality and independence of residuals, our Theorems A and B may be regarded as supporting the thesis that theory developed on the basis of (2.4) is often robust for large samples under deviations from assumption, a problem of some interest in time-series analysis. (Of course the process $\left\{X_{n}\right\}$ may be in its stationary regime, in which case (2.3) implies that $m$ is its first autocorrelation; however the distribution of residuals would still appear to be far from normal. It is relevant to note however that in its stationary regime the process $\left\{X_{n}\right\}$ has in fact its entire correlogram identical with that of the first-order autoregression.)

\section{Proof of Theorem A}

We carry out the proof in a sequence of steps, which are each of somewhat broader significance. The first step is a law of large numbers for martingales describing the effect of averaging the successive differences between each of a sequence of random variables and its best predictor on the basis of its past. This theorem partially extends a result given by Loève ((1963), p. 387).

Theorem 1. Let $\left\{\mathscr{F}_{n}, n=0,1,2, \cdots\right\}$ be an increasing sequence of $\sigma$-fields, and $\left\{U_{n}, n=0,1,2, \cdots\right\}$ a sequence of random variables, with $U_{n}$ measurable with respect to $\mathscr{F}_{n}$; and $U$ a random variable such that $E(|U|)<\infty$ and $P\left(\left|U_{n}\right|>x\right) \leqq P(|U|>x)$ for each $x \geqq 0$ and $n \geqq 0$. Then

$$
n^{-1} \sum_{k=1}^{n}\left[U_{k}-E\left(U_{k} \mid \mathscr{F}_{k-1}\right)\right] \stackrel{\mathrm{p}}{\rightarrow} 0
$$

as $n \rightarrow \infty$. If $E|U| \log ^{+}|U|<\infty$, then the convergence in probability may be strengthened to almost sure (a.s.) convergence.

Proof. Firstly we form the truncated random variables $U_{n}^{\prime}=U_{n} I\left(\left|U_{n}\right| \leqq n\right)$ and note that the sequence $\left\{U_{n}^{\prime}-E\left(U_{n}^{\prime} \mid \mathscr{F}_{n-1}\right), \mathscr{F}_{n}, n \geqq 1\right\}$ is a martingale difference sequence. We have 


$$
\begin{aligned}
\sum_{n=1}^{\infty} \frac{1}{n^{2}} E\left(U_{n}^{\prime}-E\left(U_{n}^{\prime} \mid \mathscr{F}_{n-1}\right)\right)^{2} & \leqq \sum_{n=1}^{\infty} \frac{1}{n^{2}} E\left(U_{n}^{\prime}\right)^{2} \\
& \leqq 2 \sum_{n=1}^{\infty} \frac{1}{n^{2}} \int_{0<x \leqq n} x P\left(\left|U_{n}\right|>x\right) d x \\
& \leqq 2 \sum_{n=1}^{\infty} \frac{1}{n^{2}} \int_{0<x \leqq n} x P(|U|>x) d x \\
& =2 \sum_{n=1}^{\infty} \frac{1}{n^{2}} \sum_{k=1}^{n} \int_{k-1<x \leqq k} x P(|U|>x) d x \\
& \leqq 2 \sum_{n=1}^{\infty} \frac{1}{n^{2}} \sum_{k=1}^{n} k P(|U|>k-1) \\
& =2 \sum_{k=1}^{\infty} k P(|U|>k-1) \sum_{n=k}^{\infty} \frac{1}{n^{2}} \\
& \leqq 4 \sum_{k=1}^{\infty} P(|U|>k-1)<\infty
\end{aligned}
$$

since $E(|U|)<\infty$. Thus the application of a standard martingale convergence theorem (e.g., Neveu (1965), p. 147) gives

$$
n^{-1} \sum_{k=1}^{n}\left[U_{k}^{\prime}-E\left(U_{k}^{\prime} \mid \mathscr{F}_{k-1}\right)\right] \stackrel{\text { a.s. }}{\rightarrow} 0 .
$$

Next, we note

$$
\sum_{k=1}^{\infty} P\left(U_{k} \neq U_{k}^{\prime}\right)=\sum_{k=1}^{\infty} P\left(\left|U_{k}\right|>k\right) \leqq \sum_{k=1}^{\infty} P(|U|>k)<\infty,
$$

so that the sequences $\left\{U_{k}\right\}$ and $\left\{U_{k}^{\prime}\right\}$ are tail-equivalent, and hence from (3.1)

$$
n^{-1} \sum_{k=1}^{n}\left[U_{k}-E\left(U_{k}^{\prime} \mid \mathscr{F}_{k-1}\right)\right] \stackrel{\text { a.s. }}{\rightarrow} 0 .
$$

Now,

$$
n^{-1} E\left|\sum_{k=1}^{n} E\left(U_{k} I\left(\left|U_{k}\right|>k\right) \mid \mathscr{F}_{k-1}\right)\right| \leqq n^{-1} \sum_{k=1}^{n} E\left(\left|U_{k}\right| I\left(\left|U_{k}\right|>k\right)\right) \rightarrow 0
$$

as $n \rightarrow \infty$ since

$$
E\left(\left|U_{n}\right| I\left(\left|U_{n}\right|>n\right)\right) \leqq \int_{n}^{\infty} P\left(\left|U_{n}\right|>x\right) d x \leqq \int_{n}^{\infty} P(|U|>x) d x \rightarrow 0
$$

as $n \rightarrow \infty$ so that using Markov's inequality, 


$$
n^{-1} \sum_{k=1}^{n} E\left(U_{k} I\left(\left|U_{k}\right|>k\right) \mid \mathscr{F}_{k-1}\right)=n^{-1} \sum_{k=1}^{n}\left[E\left(U_{k} \mid \mathscr{F}_{k-1}\right)-E\left(U_{k}^{\prime} \mid \mathscr{F}_{k-1}\right)\right] \stackrel{\mathrm{p}}{\rightarrow} 0
$$

and hence

$$
n^{-1} \sum_{k=1}^{n}\left[U_{k}-E\left(U_{k} \mid \mathscr{F}_{k-1}\right)\right] \stackrel{p}{\rightarrow} 0 .
$$

Furthermore, if $E|U| \log ^{+}|U|<\infty$, then

$$
\sum_{n=1}^{\infty} n^{-1} E\left(\left|U_{n}\right| I\left(\left|U_{n}\right|>n\right) \mid \mathscr{F}_{n-1}\right)<\infty \text { a.s. }
$$

since

$$
\begin{aligned}
\sum_{n=1}^{\infty} n^{-1} E & \left(E\left(\left|U_{n}\right| I\left(\left|U_{n}\right|>n\right) \mid \mathscr{F}_{n-1}\right)\right) \\
& =\sum_{n=1}^{\infty} n^{-1} E\left(\left|U_{n}\right| I\left(\left|U_{n}\right|>n\right)\right) \\
& \leqq \sum_{n=1}^{\infty} n^{-1} \int_{n}^{\infty} P\left(\left|U_{n}\right|>x\right) d x \\
& \leqq \sum_{n=1}^{\infty} n^{-1} \int_{n}^{\infty} P(|U|>x) d x \\
& =\sum_{n=1}^{\infty} n^{-1} \sum_{k=n}^{\infty} \int_{k<x \leqq k+1} P(|U|>x) d x \\
& \leqq \sum_{k=1}^{\infty} P(|U|>k) \sum_{n=1}^{k} n^{-1} \leqq \sum_{k=1}^{\infty}(1+\log k) P(|U|>k)<\infty
\end{aligned}
$$

Thus, using the Kronecker lemma

$$
n^{-1} \sum_{k=1}^{n} E\left(\left|U_{k}\right| I\left(\left|U_{k}\right|>k\right) \mid \mathscr{F}_{k-1}\right) \stackrel{\text { a.s. }}{\rightarrow} 0
$$

and hence

$n^{-1} \sum_{k=1}^{n} E\left(U_{k} I\left(\left|U_{k}\right|>k\right) \mid \mathscr{F}_{k-1}\right)=n^{-1} \sum_{k=1}^{n}\left[E\left(U_{k} \mid \mathscr{F}_{k-1}\right)-E\left(U_{k}^{\prime} \mid \mathscr{F}_{k-1}\right)\right] \stackrel{\text { a.s. }}{\rightarrow} 0$ as required. This completes the proof of the theorem.

The following result is the one which is responsible for the consistency of the estimators.

Theorem 2. Let $Y_{i}=X_{i}-\mu, i=0,1,2, \cdots$ as before. Then 
(i) $\quad \sum_{i=1}^{n} Y_{i} / n \stackrel{p}{\rightarrow} 0$;

(ii) $\sum_{i=1}^{n} Y_{i}^{2} / n \stackrel{p}{\rightarrow}\left(1-m^{2}\right)^{-1} c^{2}$;

(iii) $\sum_{i=1}^{n} Y_{i+1} Y_{i} / n \stackrel{\mathrm{p}}{\rightarrow} m\left(1-m^{2}\right)^{-1} c^{2}$.

If the offspring, immigration and initial distributions have finite third moments, then the above convergences can be replaced by almost sure convergence. (Note that if $\left\{X_{n}\right\}$ is stationary, $c^{2}=E\left(\operatorname{var}\left(Y_{n+1} \mid Y_{n}\right)\right)$ and $\left(1-m^{2}\right)^{-1} c^{2}=\operatorname{var} Y_{n}$.)

Proof. All of (i)-(iii) follow easily from three applications of Theorem 1, once the appropriate 'dominating' random variable has been found, and it is this point which takes up most of the proof.

Firstly, we note that the state space, $S$, of our process $\left\{X_{n}\right\}$, considered as a denumerable Markov chain, consists (a) of an irreducible positive recurrent set, $J^{*}$, of states including the state 0 , plus, possibly (b) 'ephemeral' states from which transition into the positive recurrent set, $J^{*}$, occurs in one step with probability one (see Seneta (1969), Section 5.2; this reference excludes the case $F(s)=\alpha+\beta s$, independent consideration of which reveals that $S=J^{*}$ ).

Thus it follows that $X_{n}=0$ infinitely often with probability one, and the time between successive returns to zero has finite mean.

Next, for our process $\left\{X_{n}\right\}$, let $T=\min \left[n: X_{n}=0\right]$ and consider $X=\sup _{0 \leqq k \leqq T}$ $X_{k}$. We clearly have $E(T)<\infty$ in view of the finiteness of the mean time between successive returns to zero and we go on to show that $E\left(X^{2}\right)<\infty$. We do this by noting that

$$
X^{2}=\sup _{0 \leqq k \leqq T} X_{k}^{2} \leqq \sum_{k=0}^{T} X_{k}^{2}
$$

and showing that $E\left(\Sigma_{k=0}^{T} X_{k}^{2}\right)<\infty$.

Now for $r=1$ or 2 ,

$$
\begin{aligned}
E\left(\sum_{k=1}^{T} X_{k}^{r}\right) & =\sum_{n=1}^{\infty} \sum_{k=1}^{n} \int_{\{T=n\}} X_{k}^{r} d P \\
& =\sum_{k=1}^{\infty} \sum_{n=k}^{\infty} \int_{\{T=n\}} X_{k}^{r} d P \\
& =\sum_{k=1}^{\infty} \int_{\{T \geqq k\}} X_{k}^{r} d P \\
& =\sum_{k=1}^{\infty} \int_{\{T \geqq k\}} E\left(X_{k}^{r} \mid \mathscr{F}_{k-1}\right) d P
\end{aligned}
$$

since $\{T \geqq k\} \in \mathscr{F}_{k-1}$, the $\sigma$-field generated by $X_{0}, \cdots, X_{k-1}$. 
First we look at the case $r=1$. We have for $k \geqq 0$,

$$
\begin{aligned}
c_{k+1} & =\int_{\{T \geqq k+1\}} E\left(X_{k+1} \mid \mathscr{F}_{k}\right) d P \\
& =\int_{\left\{T \geqq k, X_{k}>0\right\}}\left(m X_{k}+\lambda\right) d P \\
& =m \int_{\left\{T \geqq k, X_{k}>0\right\}} X_{k} d P+\lambda P(T \geqq k+1) \\
& =m \int_{\{T \geqq k\}} X_{k} d P+\lambda P(T \geqq k+1) \\
& =m c_{k}+\lambda P(T \geqq k+1)
\end{aligned}
$$

and $c_{0}=E\left(X_{0}\right)<\infty$. From the recurrence relation (3.5) we find that

and hence, using (3.4),

$$
c_{k}=m^{k} E\left(X_{0}\right)+\lambda \sum_{j=1}^{k} m^{k-j} P(T \geqq j), \quad k \geqq 1,
$$

$$
\begin{aligned}
E\left\{\sum_{k=1}^{T} X_{k}\right\}=\sum_{k=1}^{\infty} c_{k} & =\frac{m E X_{0}}{1-m}+\lambda \sum_{k=1}^{\infty} \sum_{j=1}^{k} m^{k-j} P(T \geqq j) \\
& =\frac{m E X_{0}}{1-m}+\frac{\lambda}{1-m} \sum_{j=1}^{\infty} P(T \geqq j)<\infty
\end{aligned}
$$

since $E(T)<\infty$.

We now adopt a similar approach for the case $r=2$. First note that

$$
\begin{aligned}
E\left(X_{k+1}^{2} \mid \mathscr{F}_{k}\right) & =E\left(\left(X_{k+1}-E\left(X_{k+1} \mid \mathscr{F}_{k}\right)\right)^{2} \mid \mathscr{F}_{k}\right)+\left(E\left(X_{k+1} \mid \mathscr{F}_{k}\right)\right)^{2} \\
& =X_{k} \sigma^{2}+b^{2}+\left(m X_{k}+\lambda\right)^{2}
\end{aligned}
$$

since $X_{k+1}-E\left(X_{k+1} \mid \mathscr{F}_{k}\right)$, conditional on $\mathscr{F}_{k}$, is the sum of $X_{k}+1$ independent random variables, $X_{k}$ of them having the distribution of $Z_{1}-m$ and the other the distribution of $I-\lambda$. Then, for $k \geqq 0$ and using (3.7),

$$
\begin{aligned}
d_{k+1} & =\int_{\{T \geqq k+1\}} E\left(X_{k+1}^{2} \mid \mathscr{F}_{k}\right) d P \\
& =\int_{\left\{T \geqq k, X_{k}>0\right\}}\left[X_{k} \sigma^{2}+b^{2}+\left(m X_{k}+\lambda\right)^{2}\right] d P \\
& =\int_{\{T \geqq k\}}\left[X_{k} \sigma^{2}+b^{2}+\left(m X_{k}+\lambda\right)^{2}\right] d P \\
& =m^{2} d_{k}+\left(\sigma^{2}+2 m \lambda\right) c_{k}+\left(b^{2}+\lambda^{2}\right) P(T \geqq k+1)
\end{aligned}
$$


and $d_{0}=E\left(X_{0}^{2}\right)$. From the recurrence relation (3.8) we find that

$d_{k+1}=m^{2(k+1)} E X_{0}^{2}+\left(\sigma^{2}+2 m \lambda\right) \sum_{j=0}^{k} m^{2(k-j)} c_{j}+\left(b^{2}+\lambda^{2}\right) \sum_{j=0}^{k} m^{2(k-j)} P(T \geqq j+1)$, $k \geqq 0$, and hence, using (3.4), (3.6) and $E(T)<\infty$,

$$
\begin{aligned}
E\left(\sum_{k=1}^{T} X_{k}^{2}\right)= & \sum_{k=1}^{\infty} d_{k} \\
= & \frac{m^{2} E\left(X_{0}^{2}\right)}{1-m^{2}}+\left(\sigma^{2}+2 m \lambda\right) \sum_{k=0}^{\infty} \sum_{j=0}^{k} m^{2(k-j)} c_{j} \\
& \quad+\left(b^{2}+\lambda^{2}\right) \sum_{k=0}^{\infty} \sum_{j=0}^{k} m^{2(k-j)} P(T \geqq j+1) \\
= & \frac{m^{2} E\left(X_{0}^{2}\right)}{1-m^{2}}+\frac{\sigma^{2}+2 m \lambda}{1-m^{2}} \sum_{j=0}^{\infty} c_{j}+\frac{b^{2}+\lambda^{2}}{1-m^{2}} \sum_{j=0}^{\infty} P(T \geqq j+1)<\infty .
\end{aligned}
$$

We thus find $E\left(X^{2}\right)<\infty$ as required. This completes the preliminaries and we now go on to establish the results of the theorem via three applications of Theorem 1.

Firstly, note that for any $x \geqq 0$ and $n \geqq 0, P\left(X_{n}>x\right) \leqq P(X>x)$; and of course, $E(X)<\infty$. Thus we may apply Theorem 1 with $\mathscr{F}_{k}$ the $\sigma$-field generated by $X_{0}, X_{1}, \cdots, X_{k}$ to obtain

$$
\begin{aligned}
n^{-1} \sum_{k=1}^{n}\left[Y_{k}-E\left(Y_{k} \mid \mathscr{F}_{k-1}\right)\right] & \equiv n^{-1} \sum_{k=1}^{n}\left[X_{k}-E\left(X_{k} \mid \mathscr{F}_{k-1}\right)\right] \\
& \stackrel{\mathrm{p}}{\rightarrow} 0 .
\end{aligned}
$$

Now, from (2.2),

$$
\begin{aligned}
n^{-1} \sum_{k=1}^{n}\left[Y_{k}-E\left(Y_{k} \mid \mathscr{F}_{k-1}\right)\right] & =n^{-1} \sum_{k=1}^{n}\left[Y_{k}-m Y_{k-1}\right] \\
& =n^{-1} \sum_{k=1}^{n}\left[\left(Y_{k}-m Y_{k}\right)+m\left(Y_{k}-Y_{k-1}\right)\right] \\
& =\frac{(1-m)}{n} \sum_{k=1}^{n} Y_{k}+m\left(\frac{Y_{n}-Y_{0}}{n}\right) .
\end{aligned}
$$

Thus $\sum_{k=1}^{n} Y_{k} / n \stackrel{p}{\rightarrow} 0$ since $\left(Y_{n}-Y_{0}\right) / n \stackrel{p}{\rightarrow} 0$. The last remark follows from an application of Markov's inequality since $E\left|Y_{n}-Y_{0}\right| / n \rightarrow 0$ as $n \rightarrow \infty$.

Next, note that $P\left(X_{n}^{2}>x\right) \leqq P\left(X^{2}>x\right)$ for all $n \geqq 0, x \geqq 0$, and $E\left(X^{2}\right)<\infty$. Thus by Theorem 1

$$
n^{-1} \sum_{k=1}^{n}\left[X_{k}^{2}-E\left(X_{k}^{2} \mid \mathscr{F}_{k-1}\right)\right] \stackrel{\mathrm{p}}{\rightarrow} 0,
$$


where $\mathscr{F}_{k-1}$ is the $\sigma$-field generated by $X_{0}, X_{1}, X_{2}, \cdots, X_{k-1}$, as before. Now

$$
\begin{aligned}
{\left[X_{k}^{2}-E\right.} & \left.E\left(X_{k}^{2} \mid \mathscr{F}_{k-1}\right)\right] \\
& =\left[X_{k}^{2}-\operatorname{var}\left(X_{k} \mid \mathscr{F}_{k-1}\right)-\left\{E\left(X_{k} \mid \mathscr{F}_{k-1}\right)\right\}^{2}\right] \\
& =\left[\left(Y_{k}+\mu\right)^{2}-\operatorname{var}\left(Y_{k} \mid \mathscr{F}_{k-1}\right)-\left\{E\left(Y_{k} \mid \mathscr{F}_{k-1}\right)+\mu\right\}^{2}\right] \\
& =\left[Y_{k}^{2}+\mu^{2}+2 \mu Y_{k}-\sigma^{2} Y_{k-1}-c^{2}-m^{2} Y_{k-1}^{2}-\mu^{2}-2 \mu m Y_{k-1}\right] .
\end{aligned}
$$

Thus from (3.10) and part (i) of the present Theorem

$$
n^{-1} \sum_{k=1}^{n}\left(Y_{k}^{2}-m^{2} Y_{k-1}^{2}\right)-c^{2} \stackrel{p}{\rightarrow} 0
$$

i.e.,

$$
\frac{\left(1-m^{2}\right)}{n} \sum_{k=1}^{n} Y_{k}^{2}+\frac{m^{2}}{n}\left(Y_{n}^{2}-Y_{0}^{2}\right) \stackrel{p}{\rightarrow} c^{2}
$$

and hence the result (ii) of the Theorem follows (since $Y_{n}^{2} / n \equiv\left(X_{n}^{2}-2 X_{n} \mu+\mu^{2}\right)$ $/ n \stackrel{\mathrm{p}}{\rightarrow} 0$, since $\left.E\left(X_{n}^{2} / n\right) \leqq E\left(X^{2} / n\right) \rightarrow 0\right)$.

To prove (iii), we first note that for any $x \geqq 0, n \geqq 0$

$$
P\left(\left|Y_{n} Y_{n+1}\right|>x\right) \leqq P\left(Z^{2}>x\right)
$$

where $Z$ has the distribution of $\max (X, \mu)$. Also, $E Z^{2}<\infty$ since $E X^{2}<\infty$. Thus by Theorem 1 we have

$$
n^{-1} \sum_{k=1}^{n}\left[Y_{k} Y_{k+1}-E\left(Y_{k} Y_{k+1} \mid \mathscr{F}_{k}\right)\right] \stackrel{\mathrm{p}}{\rightarrow} 0 .
$$

Now

$$
E\left(Y_{k} Y_{k+1} \mid \mathscr{F}_{k}\right)=Y_{k} E\left(Y_{k+1} \mid Y_{k}\right)
$$

by Markovicity; and by (2.2)

$$
=m Y_{k}^{2},
$$

whence

$$
n^{-1} \sum_{k=1}^{n}\left[Y_{k} Y_{k+1}-m Y_{k}^{2}\right] \stackrel{p}{\rightarrow} 0
$$

and so by part (ii) of the theorem,

$$
n^{-1} \sum_{k=1}^{n} Y_{k} Y_{k+1} \stackrel{\mathrm{p}}{\rightarrow} \frac{m}{1-m^{2}} c^{2}
$$

as required. 
This completes the proof of the convergence in probability results. Almost sure convergence under the additional conditions follows straightforwardly along similar lines. When $E\left(X_{0}^{3}\right)<\infty, E\left(Z_{1}^{3}\right)<\infty, E\left(I^{3}\right)<\infty$, it follows that $E\left(X^{3}\right)<\infty$ by an argument similar to that used to show $E(X)<\infty, E\left(X^{2}\right)<\infty$. Then, the a.s. convergence form of Theorem 1 can be used in three applications. The only other point that requires noting is that $Y_{n}^{2} / n \stackrel{\text { a.s. }}{\rightarrow} 0$ as $n \rightarrow \infty$ and this follows from the Borel-Cantelli lemma since $Y_{n}^{2} / n=\left(X_{n}^{2}-2 X_{n} \mu+\mu^{2}\right) / n$ and for any $\varepsilon>0$

$$
\sum_{n=1}^{\infty} P\left(X_{n}^{i}>n \varepsilon\right) \leqq \sum_{n=1}^{\infty} P\left(X^{i}>n \varepsilon\right)<\infty
$$

since $E\left(X^{i}\right)<\infty, i=1,2$.

Remark. It is not difficult to see that the above proof of part (i) holds without the assumptions of finite variances for the initial, offspring, and immigration distributions.

The proof of Theorem A now follows easily. That $C_{n} \stackrel{\mathbf{p}}{\rightarrow} \mu$, is just a restatement of Theorem 2, (i). Let us turn to $D_{n}$ :

$$
\begin{aligned}
n^{-1} \sum_{i=1}^{n} \hat{Y}_{i+1} \hat{Y}_{i} & =n^{-1} \sum_{i=1}^{n}\left(Y_{i+1}+\mu-C_{n}\right)\left(Y_{i}+\mu-C_{n}\right) \\
& =n^{-1} \sum_{i=1}^{n} Y_{i+1} Y_{i}+R_{n},
\end{aligned}
$$

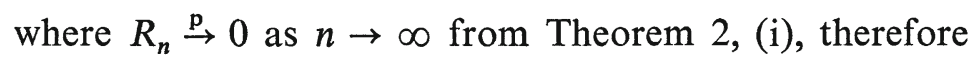

$$
n^{-1} \sum_{i=1}^{n} \hat{Y}_{i+1} \hat{Y}_{i} \stackrel{\mathrm{p}}{\rightarrow} m\left(1-m^{2}\right)^{-1} c^{2}
$$

from part (iii) of the same theorem. Similarly

$$
n^{-1} \sum_{i=1}^{n} \hat{Y}_{i}^{2} \stackrel{p}{\rightarrow}\left(1-m^{2}\right)^{-1} c^{2}
$$

from Theorem 2, parts (i) and (ii). The result of Theorem A follows immediately.

Remarks. (1) As a 'bonus' result, the above provides a consistent estimator of the variance of the limiting-stationary distribution of $\left\{X_{n}\right\}$ viz. $\left(1-m^{2}\right)^{-1} c^{2}$. This is clearly the 'obvious' one

$$
E_{n}=\sum_{i=1}^{n} \hat{Y}_{i}^{2} / n
$$


(2) The estimator

$$
\begin{aligned}
D_{n}^{*} & =1-\frac{1}{2} \frac{\sum_{i=1}^{n}\left(X_{i+1}-X_{i}\right)^{2}}{\sum_{i=1}^{n}\left(X_{t}-\bar{X}\right)^{2}} \\
& =1-\frac{1}{2} \frac{\sum_{i=1}^{n}\left(Y_{i+1}-Y_{i}\right)^{2}}{\sum_{i=1}^{n} \hat{Y}_{i}^{2}} \\
& =1-\frac{1}{2 n}\left(\sum_{i=1}^{n} Y_{i+1}^{2}+\sum_{i=1}^{n} Y_{i}^{2}-2 \sum_{i=1}^{n} Y_{i+1} Y_{i}\right) / E_{n} \\
& =\left(\sum_{i=1}^{n} Y_{i+1} Y_{i} / n\right) / E_{n}+\bar{R}_{n},
\end{aligned}
$$

where $\bar{R}_{n} \stackrel{p}{\rightarrow} 0$ as $n \rightarrow \infty$, by Theorem 2 and (3.13) so that

$$
D_{n}^{*} \stackrel{\mathrm{p}}{\rightarrow} m .
$$

Thus $D_{n}^{*}$ is also consistent for $m$.

\section{Proof of Theorem $B$}

We proceed in the manner of Section 3, first proving the following result.

Theorem 3.

$$
\lim _{n \rightarrow \infty} P\left(\alpha^{-1} \sqrt{ } n\left(\sum_{\imath=1}^{n} Y_{i} / n\right) \leqq x\right)=\Phi(x) .
$$

If, in addition to the basic assumptions of Section 1, all of the offspring, immigration and initial distributions have finite fourth moments, then

$$
\lim _{n \rightarrow \infty} P\left(\frac{\sum_{i=1}^{n} Y_{i}\left(Y_{i+1}-m Y_{i}\right)}{B \sqrt{ } n} \leqq x\right)=\Phi(x),
$$

where $B$ is a constant defined in the statement of Theorem B.

Proof. The results are obtained by making use of a central limit theorem for martingales due to Brown (1971).

First write $W_{i}=Y_{i}-E\left(Y_{i} \mid \mathscr{F}_{1-1}\right), i=1,2, \cdots$ and $S_{n}=\sum_{i=1}^{n} W_{i}$, noting that $\left\{S_{n}, \mathscr{F}_{n}, n=1,2, \cdots\right\}$ is a zero-mean martingale. Let

$$
V_{n}^{2}=\sum_{j=1}^{n} E\left(W_{j}^{2} \mid \mathscr{F}_{j-1}\right), s_{n}^{2}=E\left(V_{n}^{2}\right)=E\left(S_{n}^{2}\right)
$$

Then

$$
V_{n}^{2}=\sum_{j=1}^{n}\left(\sigma^{2} Y_{j-1}+c^{2}\right)=\sigma^{2} \sum_{j=0}^{n-1} Y_{j}+n c^{2}
$$


and

$$
\begin{aligned}
s_{n}^{2} & =\sigma^{2} \sum_{j=0}^{n-1}\left\{m^{j} E\left(Y_{0}\right)\right\}+n c^{2} \\
& =\sigma^{2} E\left(Y_{0}\right) \sum_{j=0}^{n-1} m^{j}+n c^{2} \\
& \sim n c^{2} \quad \text { as } n \rightarrow \infty .
\end{aligned}
$$

Moreover, since $V_{n}^{2}=\sigma^{2} \sum_{j=0}^{n-1} Y_{j}+n c^{2}$, it follows from Theorem 2(i) that $V_{n}^{2} / s_{n}^{2} \stackrel{\mathrm{p}}{\rightarrow} 1$ as $n \rightarrow \infty$.

Now, let $U(x)=x(1+x)^{-1}$; then

$$
0 \leqq \frac{1}{n} \sum_{j=1}^{n} W_{j}^{2} U\left(\left|W_{j}\right| \varepsilon^{-1} s_{n}^{-1}\right) \leqq \frac{1}{\varepsilon n S_{n}} \sum_{j=1}^{n}\left|W_{j}\right|^{3}
$$

Now, using Theorem 2 we see that $n^{-1} \quad \sum_{j=1}^{n} W_{j}^{2}$ converges in probability to a finite constant. Also, using the Borel-Cantelli lemma, $n^{-1} W_{n}^{2} \stackrel{\text { a.s. }}{\rightarrow} 0$ and hence $n^{-\frac{1}{2}} \sup _{1 \leqq k \leqq n}\left|W_{k}\right| \stackrel{\text { a.s. }}{\rightarrow} 0$. It then follows that

$$
n^{-3 / 2} \sum_{j=1}^{n}\left|W_{j}\right|^{3} \leqq\left(n^{-1} \sum_{j=1}^{n} W_{j}^{2}\right)\left(n^{-\frac{1}{2}} \sup _{1 \leqq k \leqq n}\left|W_{k}\right|\right) \stackrel{\mathrm{p}}{\rightarrow} 0
$$

and hence by (4.1), (4.2) and an application of Theorem 1,

$$
n^{-1} \sum_{j=1}^{n} E\left(W_{j}^{2} U\left(\left|W_{j}\right| \varepsilon^{-1} s_{n}^{-1}\right) \mid \mathscr{F}_{j-1}\right) \stackrel{\mathrm{p}}{\rightarrow} 0 .
$$

It therefore follows from Lemma 2 of Brown (1971), and the fact that $s_{n}^{-2} V_{n}^{2} \stackrel{p}{\rightarrow} 1$, that Theorem 2 of the same reference is applicable and hence

$$
\lim _{n \rightarrow \infty} P\left(s_{n}^{-1} S_{n} \leqq x\right)=\Phi(x)
$$

The result of the first part of the theoren is then immediate ifrom (4.1) and the fact that

$$
S_{n}=(1-m) \sum_{k=1}^{n} Y_{k}+m\left(Y_{n}-Y_{0}\right)
$$

The proof of the second part of the theorem follows the same pattern as the first. Write $\zeta_{i}=Y_{i}\left(Y_{i+1}-m Y_{i}\right), i=0,1,2, \cdots$ and $T_{n}=\sum_{i=1}^{n} \zeta_{i}$, noting that $\left\{T_{n}, \mathscr{F}_{n+1}, n=1,2, \cdots\right\}$ is a zero-mean martingale.

Let

Then

$$
V_{n}^{*^{2}}=\sum_{j=1}^{n} E\left(\zeta_{j}^{2} \mid \mathscr{F}_{J}\right), s_{n}^{*^{2}}=E\left(V_{n}^{*^{2}}\right)=E\left(T_{n}^{2}\right)
$$




$$
\begin{aligned}
V_{n}^{*^{2}} & =\sum_{j=1}^{n} Y_{j}^{2} E\left\{\left(Y_{j+1}-m Y_{j}\right)^{2} \mid \mathscr{F}_{j}\right\} \\
& =\sum_{j=1}^{n} Y_{j}^{2} \operatorname{var}\left(Y_{j+1} \mid \mathscr{F}_{j}\right) \\
& =\sum_{j=1}^{n} Y_{j}^{2}\left[\sigma^{2} Y_{j}+c^{2}\right]
\end{aligned}
$$

i.e.,

$$
V_{n}^{*^{2}}=c^{2} \sum_{j=1}^{n} Y_{j}^{2}+\sigma^{2} \sum_{j=1}^{n} Y_{j}^{3} .
$$

Now, a straightforward but tedious calculation shows that

$$
n^{-1} s_{n}^{*^{2}} \rightarrow \gamma \sigma^{2}+c^{4}\left(1-m^{2}\right)^{-1},
$$

where

$$
\gamma=\frac{1}{1-m^{3}}\left[E(I-\lambda)^{3}+\mu E\left(Z_{1}-m\right)^{3}+\frac{3 m c^{2}}{1-m^{2}}\right],
$$

(and is $E\left(Y_{n}^{3}\right)$ when $Y_{n}$ has its 'limiting-stationary' distribution).

Furthermore, when $E\left(X_{0}^{4}\right)<\infty, E\left(Z_{1}^{4}\right)<\infty, E\left(I^{4}\right)<\infty$, as is the case at present, it follows using the methods developed in the proof of Theorem 2, that $E\left(X^{4}\right)<\infty$. Thus we can certainly apply Theorem 1 to obtain

$$
n^{-1} \sum_{j=1}^{n}\left[Y_{j}^{3}-E\left(Y_{j}^{3} \mid \mathscr{F}_{j-1}\right)\right] \stackrel{\mathrm{p}}{\rightarrow} 0
$$

from which, and more straightforward but tedious analysis,

$$
n^{-1} \sum_{j=1}^{n} Y_{j}^{3} \stackrel{p}{\rightarrow} \gamma
$$

It therefore follows from (ii) of Theorem 2, (4.3), (4.4) and (4.5) that $s_{n}^{*-2} V_{n}^{* 2} \stackrel{\mathfrak{p}}{\rightarrow} 1$ as $n \rightarrow \infty$.

Next, again let $U(x)=x /\{1+x\}$ so that

$$
0 \leqq \frac{1}{n} \sum_{j=1}^{n} \zeta_{j}^{2} U\left(\left|\zeta_{j}\right| \varepsilon^{-1} s_{n}^{*-1}\right) \leqq \frac{1}{\varepsilon n s_{n}^{*}} \sum_{j=1}^{n}\left|\zeta_{j}\right|^{3} .
$$

Since $E\left(X^{4}\right)<\infty$ we can apply Theorem 1 to obtain

$$
n^{-1} \sum_{j=1}^{n}\left[\zeta_{j}^{2}-E\left(\zeta_{j}^{2} \mid \mathscr{F}_{j}\right)\right] \stackrel{\mathrm{p}}{\rightarrow} 0
$$

which gives, in view of (4.4), 


$$
n^{-1} \sum_{j=1}^{n} \zeta_{j}^{2} \stackrel{\mathrm{p}}{\rightarrow} \gamma \sigma^{2}+c^{4}\left(1-m^{2}\right)^{-1}
$$

Thus, as in the proof of the first part of the theorem, we have from the BorelCantelli lemma that $n^{-1} \zeta_{n}^{2} \stackrel{\text { a.s. }}{\rightarrow} 0$ so that $n^{-\frac{1}{2}} \sup _{1 \leqq k \leqq n}\left|\zeta_{k}\right| \stackrel{\text { a.s. }}{\rightarrow} 0$ and

$$
n^{-\frac{3}{2}} \sum_{j=1}^{n}\left|\zeta_{j}\right|^{3} \leqq\left(n^{-1} \sum_{j=1}^{n} \zeta_{j}^{2}\right)\left(n^{-\frac{1}{2}} \sup _{1 \leqq j \leqq n}\left|\zeta_{j}\right|\right) \stackrel{\mathrm{p}}{\rightarrow} 0 .
$$

Consequently, from (4.4), (4.6) and an application of Theorem 1,

$$
n^{-1} \sum_{j=1}^{n} E\left(\zeta_{j}^{2} U\left(\left|\zeta_{j}\right| \varepsilon^{-1} s_{n}^{*-1}\right) \mid \mathscr{F}_{j-1}\right) \stackrel{\mathrm{p}}{\rightarrow} 0
$$

We have thus again completed the formalities necessary to apply Theorem 2 of Brown (1971). This gives

$$
\lim _{n \rightarrow \infty} P\left(s_{n}^{*-1} T_{n} \leqq x\right)=\Phi(x)
$$

which provides the desired result.

Remarks. (1) Theorem 3 is given in the above setting in the interests of clarity of exposition; much more actually holds. In fact, invariance principles for suitably defined random functions in $C[0,1]$ hold for both parts of the theorem. This is a consequence of the fact that the conditions of Theorem 3 of Brown (1971) are satisfied in both parts of the theorem. The interested reader is referred to Brown's paper for details of the setting.

(2) The result of the first part of our Theorem 3 has been obtained by Pakes (1971), who uses classical results on sums of independent random variables.

The proof of Theorem B is now immediate. The first part is just the first part of Theorem 3. For the second part note that

$$
n^{-1} \sum_{i=1}^{n} \hat{Y}_{i}\left(\hat{Y}_{i+1}-m \hat{Y}_{i}\right)=n^{-1} \sum_{i=1}^{n} \hat{Y}_{i} \hat{Y}_{i+1}-m n^{-1} \sum_{i=1}^{n} \hat{Y}_{i}^{2}
$$

so that, as in the argument leading to (3.12) and (3.13),

$$
\begin{aligned}
& =n^{-1} \sum_{i=1}^{n} Y_{i} Y_{i+1}-m n^{-1} \sum_{i=1}^{n} Y_{i}^{2}+\overline{\bar{R}}_{n} \\
& =n^{-1} \sum_{i=1}^{n} Y_{i}\left(Y_{i+1}-m Y_{i}\right)+\overline{\bar{R}}_{n},
\end{aligned}
$$

where $\sqrt{ } n \overline{\bar{R}}_{n} \stackrel{\mathrm{p}}{\rightarrow} 0$ as $n \rightarrow \infty$ in view of part (i) of Theorem 2 and the first part of Theorem 3, and the second part follows from this, (3.13), and the second part of Theorem 3. 
Remarks. (1) The values $\alpha^{2} / n$ and $\beta^{2} / n$ give an indication of the asymptotic decline of the variances of the estimators $C_{n}$ and $D_{n}$ respectively; this may be of importance in practical considerations, and we shall return to it in the next section.

(2) It can be seen without difficulty from the kind of discussion given at the conclusion of Section 3, and above, that $D_{n}^{*}$ may be inserted in place of $D_{n}$ in Theorem B.

\section{Analysis of historical development}

The simple Galton-Watson process with immigration, where $F(s)=1-m+m s$ and $B(s)=\exp \{\lambda(1-s)\}$, in its stationary state, was developed as early as 1915 by Smoluchowski (for an account of this early work see Chapter III of the article of Chandrasekhar (1943)). It was used as a model for the fluctuation in the number of particles contained in a geometrically well-defined small element of volume $v$, in a much larger volume of solution containing particles exhibiting random motion, the system being in equilibrium, and observations on $v$ being made at constant intervals of time apart. The number $P=1-m$ was called the 'probability after-effect factor', and, of course (as also $\lambda$ ) depends on the precise circumstances of the problem.

Moreover, it was noted from the above physical situation, and can be easily deduced analytically, that the stationary distribution is also Poisson, with p.g.f. $\exp \{\mu(1-s)\}, \mu P=\lambda$. The quantity of interest was in fact the number $P$, which was to be estimated from the experimental data. The method used was to note first that (since the regime is stationary, and the stationary distribution is Poisson)

$$
E\left(\left(X_{n+1}-X_{n}\right)^{2}\right)=2 \mu P .
$$

Then from a long series of observations on the sequence $\left\{X_{n}\right\}, C_{n}=\bar{X}$ was found, and $\mu$ (the variance as well as the mean of the stationary distribution) was estimated by $\sum_{i=1}^{n} \hat{Y}_{i}^{2} / n$, and $E\left(\left(X_{n+1}-X_{n}\right)^{2}\right)$ by $\sum_{i=1}^{n}\left(X_{i+1}-X_{i}\right)^{2} / n$, which led to the estimate $\hat{P}_{1}$ of $P$ :

$$
\hat{P}_{1} \equiv \frac{1}{2} \frac{\sum_{i=1}^{n}\left(X_{i+1}-X_{i}\right)^{2}}{\sum_{i=1}^{n}\left(X_{i}-\bar{X}\right)^{2}}=1-D_{n}^{*},
$$

so that in fact $m=1-P$ is estimated by our estimator $D_{n}^{*}$.

Once $P$ has been estimated statistically, its value may be compared with that obtained analytically on the basis of the parameters of the system. Experimental verification on Smoluchowski's theory using colloidal solutions was carried out by Westgren ((1916), (1918)) in particular, on this basis; the agreement was found to be very good. Another early experiment performed on the basis of Smoluchowski's theory was that of Fürth ((1918), (1919)) (whose data we shall use 
in the next section). The physical context was different; systematic counts were made of the number of pedestrians in a block every five seconds (a short enough time interval for any pedestrian to be observed at least twice with a large probability).

It is clear that the estimation of $P$ may also in fact be used to estimate parameters of the system, and this fact is used in Westgren's work to determine Avogadro's constant (and could be used to estimate the 'average speed of a pedestrian' in Fürth's context, as Chandrasekhar points out).

Also, it is necessary to mention that, whereas recourse to the very early memoirs reveals that in fact the estimator $\hat{P}_{1}$ was used to estimate $P$, Chandrasekhar's account of the Smoluchowski theory suggests that in fact $\mu$ in (5.1) was actually estimated by $\bar{X}$, which in turn leads to the estimator of $P$ :

$$
\hat{P}_{2}=\frac{1}{2} \frac{\sum_{i=1}^{n}\left(X_{i+1}-X_{i}\right)^{2}}{n \bar{X}},
$$

so that $m$ would be estimated by

$$
.1-\hat{P}_{2}=1-\frac{1}{2} \frac{\sum_{i=1}^{n}\left(X_{i+1}-X_{i}\right)^{2}}{n X},
$$

which is, of course, also consistent for $m$, in this special situation, since $\bar{X}$ is also consistent for the variance $\mu$ of the stationary-distribution. (Fürth in fact used the estimator (5.3), as an analysis of his data reveals.)

These last two paragraphs are particularly relevant to a more recent application of Smoluchowski's theory, by Rothschild (1953) who (under the influence of Chandrasekhar's account) uses the estimator (5.3) in the estimation of sperm speeds from counts of successive numbers in cinemicrographs. Rothschild's paper brings out several other features not noticeable in the earlier literature, for firstly he notes that previous authors paid little attention to the precision of the estimation method, and presents an unproved formula for the variance, obtained from moments, up to the fourth, of $\left\{X_{n}\right\}$. According to an account in Bartlett ((1955), p. 248) this translates as

$$
\operatorname{var}\left(1-\hat{P}_{2}\right) \sim\left\{(1-m)^{2}(3+m)(1+m)^{-1}+m(1-m) \mu^{-1}\right\} / n,
$$

which is of the same order of decline as our formula $\beta^{2} / n$, although we are not aware as to the nature of the approximations used in the derivation. Secondly, Rothschild's account emphasizes the fact that the process is actually occurring in continuous time, although (even with a movie camera, as in his experiments) observations are intermittent; and estimation is therefore for the discrete skeleton of the process. One consequence of this is that the choice of interval between observations is important for the branching assumptions to be more closely satisfied. A second is that in such a situation it is necessary to translate the esti- 
mates from the discrete to continuous time context, which requires a knowledge of the relationship between the process and its discrete skeleton. We take up this point in the next paragraph.

A maximum-likelihood approach to the problem of estimating $m$ and $\mu$ in the simple Smoluchowski model (but dropping the assumption of stationary regime) was made by Bartlett and his student Patankar (see Bartlett (1955), p. 246-247). The discussion begins with the more fundamental continuous-time Markov branching process with immigration (underlying the Smochulowski model), which has p.g.f.

$$
E\left\{s^{X(t)} \mid X(0)=k\right\}=\left[1+e^{-v t}(s-1)\right]^{k} \exp \left\{\mu\left(1-e^{-v t}\right)(s-1)\right\} .
$$

Observations on this process are made at unit time intervals (w.l.o.g.), so that in fact one observes the process $\left\{X_{n}\right\}$ with p.g.f.

$$
E\left\{s^{X_{n}} \mid X_{0}=k\right\}=\left[1+m^{n}(s-1)\right]^{k} \exp \left\{\mu\left(1-m^{n}\right)(s-1)\right\},
$$

where $m=e^{-v}$. It is easy to check that $\left\{X_{n}\right\}$ corresponds to a Galton-Watson process with immigration having offspring and immigration p.g.f.'s

$$
\begin{aligned}
& F(s)=(1-m)+m s, \\
& B(s)=\exp \{\lambda(s-1)\},
\end{aligned}
$$

where $\lambda=\mu(1-m)$, as before, so that the parameters $\mu$ and $\nu$ of the continuoustime process are in fact validly estimated from the techniques mentioned before. The method of maximum likelihood then yields the estimator $\hat{\mu}=\sum_{i=1}^{n} X_{i} / n$, but the other maximum-likelihood estimator, for $m$, does not emerge in useful form.

In general where the offspring and immigration distributions are suitably parametrized as in the above simple model, an approach to the estimation problem is possible using the method of maximum likelihood. Such an approach has theoretical advantages, insofar as one is able to make use of the general theory of maximum likelihood estimation in the Markov chain context (e.g., Billingsley (1961)). However, the few other examples investigated indicate that difficulties similar to the above case arise in the estimation of $m$.

In conclusion to this historical discussion, we mention that several other physical models making use of the Galton-Watson process with immigration are mentioned in Chapter 5 of Seneta (1969).

\section{Some complements}

(a) The discrete skeleton of a Markov branching process with immigration. We shall show that if a regular homogeneous continuous-time Markov branching process with immigration, $\{X(t)\}$, is observed at equally spaced instants of time, 
we obtain a Galton-Watson process with immigration. We shall consider unit time intervals for simplicity of exposition.

Let $\Phi(t ; s)$ be the p.g.f. of a regular Markov branching process initiated by a single individual (ignoring immigration) i.e., the p.g.f. of the direct descendants, at time $t$. Let $\delta_{k 0}+q_{k} \Delta t+o(\Delta t)$ be the probability of $k$ immigrants in time interval $\Delta t$, so that $g(1)=\sum_{k=0}^{\infty} q_{k}=0$, where $g(s)=\sum_{k=0}^{\infty} q_{k} s^{k}$. Let us consider, further, a process with immigration $\{X(t)\}$ initiated by a fixed number, $i \geqq 0$, of individuals (extension to an initial distribution is trivial). Then

$$
P_{i}(t ; s) \equiv E\left[s^{X(t)} \mid X(0)=i\right]=\Phi^{i}(t ; s) A(t ; s),
$$

where Sevast'yanov (1957) has shown that

$$
A(t ; s)=E\left[s^{X(t)} \mid X(0)=0\right]=\exp \left\{\int_{0}^{t} g[\Phi(u ; s)] d u\right\} .
$$

Now, it is well known that the discrete skeleton of an ordinary Markov branching process (without immigration) is a Galton-Watson process, so that $\Phi(n ; s)=\Phi_{n}(1 ; s)$, where $\Phi_{n}(1 ; s)$ is the $n$th functional iterate of $\Phi(1 ; s)$. We know in fact that, more generally, for any $u, v>0$, the characteristic branching property implies that $\Phi(u ; \Phi(v ; s))=\Phi(u+v ; s)$.

Thus, if we put $X(n)=X_{n}$, we have

$$
E\left\{s^{X_{n+1}} \mid X_{0}=i\right\}=P_{i}(n+1 ; s)=\Phi^{i}(n ; \Phi(1 ; s)) A(n+1 ; s)
$$

since

$$
\Phi(n+1 ; s)=\Phi_{n+1}(1 ; s)=\Phi_{n}(1 ; \Phi(1 ; s))=\Phi(n ; \Phi(1 ; s)) .
$$

Now, consider the following argument:

$$
\begin{aligned}
A(1 ; s) A(n ; \Phi(1 ; s)) & =\exp \left\{\int_{0}^{1} g[\Phi(u ; s)] d u\right\} \exp \left\{\int_{0}^{n} g[\Phi(u ; \Phi(1 ; s))] d u\right\} \\
& =\exp \left\{\int_{0}^{1} g[\Phi(u ; s)] d u\right\} \exp \left\{\int_{0}^{n} g[\Phi(u+1 ; s)] d u\right\} \\
& =\exp \left\{\int_{0}^{1} g[\Phi(u ; s)] d u\right\} \exp \left\{\int_{1}^{n+1} g[\Phi(u ; s)] d u\right\} \\
& =\exp \left\{\int_{0}^{n+1} g[\Phi(u ; s)] d u\right\},
\end{aligned}
$$

i.e.,

$$
A(1 ; s) A(n ; \Phi(1 ; s))=A(n+1 ; s),
$$

so that (6.1) yields 


$$
P_{i}(n+1 ; s)=A(1 ; s) P_{i}(n ; \Phi(1 ; s))
$$

which is the evolutionary equation of a Galton-Watson process with immigration, as required, with offspring p.g.f. $F(s) \equiv \Phi(1 ; s)$, as expected, and immigration p.g.f. of rather complex form

$$
B(s) \equiv A(1 ; s)=\exp \left\{\int_{0}^{1} g[\Phi(u ; s)] d u\right\} .
$$

Henceforth we assume the existence of first and second derivatives at $s=1-$ of the continuous-time immigration and offspring generating functions, $g(s)$ and $k(s)$. Let us put in particular $k^{\prime}(1-)=-v$ for the mean offspring rate, assuming $v>0$ for a subcritical process; and put $\tau \equiv g^{\prime}(1-)$ for the immigration rate.

It follows that using our general results we are able to estimate from the process $\left\{X_{n}\right\}$ the mean $\mu$ of the limiting-stationary distribution (the same for the continuous-time process and its discrete skeleton), and the decay parameter $v$ where $e^{-v}=F^{\prime}(1-)=m$.

To estimate the immigration intensity, we note that, differentiating (6.2) with respect to $s$ gives

$$
B^{\prime}(s)=\left\{\int_{0}^{1} \frac{d(g[\Phi(u ; s)]}{d s} d u\right\} B(s),
$$

differentiation under the integral at $s \in(0,1)$ being permissible. Let $s \rightarrow 1-$; we have by dominated convergence

$$
\lambda \equiv B^{\prime}(1-)=g^{\prime}(1-) \int_{0}^{1} \Phi^{\prime}(u ; 1) d u=\tau v^{-1}\left(1-e^{-v}\right)
$$

since $\Phi^{\prime}(u ; 1)=[d \Phi(u ; s) / d s]_{s=1}=e^{-v \mu}$. Thus

$$
\tau v^{-1}=\lambda\left(1-e^{-v}\right)^{-1}=\lambda(1-m)^{-1}=\mu,
$$

so that $\tau=v \mu$ and a consistent estimator for $\tau$ is obtained by multiplying two consistent estimators, of $v$ and $\mu$ respectively.

Clearly this discussion subsumes that at the conclusion of Section 5 .

Clearly also, Theorem B is usable in drawing inferences about $\mu$, and about $\nu$, so long as the constants $\alpha, \beta$ can be determined. This can in fact be done with the aid of the generators $k(s)$ and $g(s)$. Thus the moments corresponding to $F(s) \equiv \Phi(1 ; s)$ may be obtained from a knowledge of $k(s)$ as indicated in Harris ((1963), p. 103). Those corresponding to $B(s)=A(1 ; s)$ can be obtained in an analogous manner involving both $k(s)$ and $g(s)$; or from differentiation of (6.2) using $g(s)$ and the known moments of $\Phi(u ; s)$. (The deduction of (6.3) is an example.) 
(b) A numerical example. Below we give numerical values of estimators obtained for the Smoluchowski model, using Fürth's data of 505 readings (observation stopped with 506th reading zero) (Fürth (1918). Tabelle 1). For this specific model, there are two estimators for $\mu$ and three for $m$ on the basis of the foregoing discussion. This leads to six possible estimators for $\lambda$, from $\lambda \equiv \mu(1-m)$. The $\lambda$ values are given in the body of the table.

(Westgren's (1916) data are much more extensive; for example, Versuchsreihe C, pp. 12-13, contains 1583 readings. However, Versuchsreihe C, as also his other tables, contains numerous breaks, and the longest run, occurring in Versuchsreihe $\mathrm{A}$ is itself of about 480 readings, with one number missing. His analysis more or less ignores the fact that he is therefore dealing in each experimental circumstance with several realizations, and his estimation procedure is not purely statistical, 'true' values being partially used. Neither difficulty arises in Fürth's circumstances.)

$$
n=505, \sum_{i=1}^{n}\left(X_{i+1}-X_{i}\right)^{2} / n=1.006
$$

\begin{tabular}{|c|c|c|c|}
\hline$m$ & $\begin{array}{c}D_{n} \\
0.666\end{array}$ & $\begin{array}{c}D_{n}^{*} \\
0.666\end{array}$ & $\begin{array}{r}1-\hat{P}_{2} \\
0.684\end{array}$ \\
\hline $\begin{array}{c}\bar{X} \\
1.592\end{array}$ & 0.532 & 0.532 & 0.503 \\
\hline $\begin{array}{l}\sum_{i=1}^{n}\left(X_{i}-\bar{X}\right)^{2} / n \\
1.505\end{array}$ & 0.503 & 0.503 & 0.476 \\
\hline
\end{tabular}

\section{References}

Bartlett, M. S. (1955) An Introduction to Stochastic Processes. Cambridge University Press, Cambridge.

Billingsley, P. (1961) Statistical Inference for Markov Processes. University of Chicago Press, Chicago.

Brown, B. M. (1971) Martingale central limit theorems. Ann. Math. Statist. 42, 59-66.

Chandrasekhar, S. (1943) Stochastic problems in physics and astronomy. Rev. Mod. Phys. 15, 1-89. (Also in Selected Papers on Noise and Stochastic Processes, ed. N. Wax, Dover, New York, 1954.)

FÜRTH, R. (1918) Statistik und Wahrscheinlichkeitsnachwirkung. Z. Physik. 19, 421-426; ibid. 20 (1919) 21.

Hannan, E. J. (1960) Time Series Analysis. Methuen, London.

HARris, T. E. (1963) The Theory of Branching Processes. Springer, Berlin.

Heatнсоте, C. R. (1966)'Corrections and comments on the paper "A branching process allowing immigration". J. R. Statist. Soc. B 28, 213-217. 
HeYde, C. C. (1971) Some central limit analogues for supercritical Galton-Watson processes. J. Appl. Prob. 8, 52-59.

Heyde, C. C. AND Sente, E. (1971) Analogues of classical limit theorems for the supercritical Galton-Watson process with immigration. Math. Biosciences 11, 249-259.

Lok̀ve, M. (1963) Probability Theory. 3rd Ed. Van Nostrand, New York.

Neveu, J. (1965) Mathematical Foundations of the Calculus of Probability. Holden-Day, San Francisco.

Pakes, A. G. (1971) Branching processes with immigration. J. Appl. Prob. 8, 32-42.

RotHSCHILD, Lord (1953) A new method of measuring the activity of spermatozoa. J. Exp. Biol. 30, 178-199. $1-42$.

Seneta, E. (1969) Functional equations and the Galton-Watson process. Adv. Appl. Prob. 1,

Sevast'yanov, B. A. (1957) Limit theorems for branching random processes of special form (in Russian). Teor. Veroyat. Primenen. 2, 339-348.

Westgren, A. (1916) Die Veränderungsgeschwindigkeit der lokalen Teilchenkonzentration in kolloiden Systemen. (Erste Mitteilung). Ark. Mat. Astronom. Fys. Band 11, No. 14, 24 pp. (Zweite Mitteilung: 1918; Band 13, No. 14, 18 pp.). 